\title{
A Standard Mission Profile for Hybrid-Electric Regional Aircraft based on Web Flight Data
}

\author{
$1^{\text {st }}$ Benedict Jux \\ Inst. of Electrical Engineering (ETI) \\ Karlsruhe Institut of Technology (KIT) \\ Karlsruhe, Germany \\ benedict.jux@kit.edu
}

\author{
$2^{\text {nd }}$ Simon Foitzik \\ Inst. of Electrical Engineering (ETI) \\ Karlsruhe Institut of Technology (KIT) \\ Karlsruhe, Germany \\ simon.foitzik@kit.edu
}

\author{
$3^{\text {rd }}$ Martin Doppelbauer \\ Inst. of Electrical Engineering (ETI) \\ Karlsruhe Institut of Technology (KIT) \\ Karlsruhe, Germany \\ martin.doppelbauer@kit.edu
}

\begin{abstract}
To reduce emissions of air traffic, future aircraft will install hybrid-electric propulsion systems. We require the load conditions over the time in service, to design these aircraft. In this paper, we propose a mission profile for a regional aircraft with a hybrid-electric propulsion system. We focused on regional aircraft, which are in service in Ireland and the United Kingdom (UK). The reference aircraft ATR 72-600 is a turboprop aircraft with a capacity of 70 passengers. To propose a mission profile, we have analyzed more than 6000 flights of four different aircraft of the same type. Input data is provided by online databases, which collect flight data. We are able to show that the maximum available power is needed for about 52 seconds during takeoff and climb phase of the flight. The median flight time is 59 minutes and 30 seconds. The average required power is $53 \%$ of the maximum power. The average traveled distance is $407 \mathrm{~km}$, which is less than one third of the aircraft's maximum reach. These findings are needed for calculating the lifetime of drivetrain components of a hybrid or all electric aircraft. In our further work, we will design an electric machine for regional aircraft. This mission profile will be used to design different power train components.
\end{abstract}

Index Terms-Electric aircraft, Mission Profile, Electric Machines, ADS-B

\section{INTRODUCTION}

Safety aspects in aerospace engineering are most critical during the design process. In the design, we face conflicting objectives. Making the aircraft efficient requires that the aircraft is as light as possible [1]. The stresses on drivetrain components are primarily from power requirements over time. On the mechanical side, for example, these are torque, vibrations or ambient influences. In electric machines for example, the aging of the winding system due to high temperature often leads to a failure of the machine [2], [3]. Only generic mission profiles are available for conventional aircraft [1] and hybridelectric aircraft [4]. We generate more detailed mission profiles with web services providing flight data. These services collect the transmitted data from aircraft, which frequently broadcast their flight information through the Automatic Dependent Surveillance-Broadcast (ADS-B). This system is used by the traffic control to manage the air traffic [5]. In this work we focus on four regional aircraft of the type ATR 72-600 which are operated by Aer Lingus Regional in Ireland and the UK.

\section{THEORY}

\section{A. Robust Design}

The conflicting objectives weight, efficiency and safety, lead to higher component stresses and therefore to a higher risk of failure [1]. One method for robust desgin, which is proposed by the German Electrical and Electronic Manufacturers' Association (ZVEI), is shown in Fig. 1. The flow diagram is simplified, to show the basic steps of a robust design. Step (1) in the validation process determines and defines the application, which in our case is a hybrid-electric regional aircraft. Step (2) is to define the application mission profile. This profile will be proposed in this paper. In the further design process of the different drivetrain components, we must identify the key risks and failure mechanisms (3) and also validate them (4),(5). These are the main topics, which need to be focused on during the design process.

\section{B. Failure of Electric Machines}

Since our focus is on electric machines, we will identify the main key risks and failure mechanisms for an electric

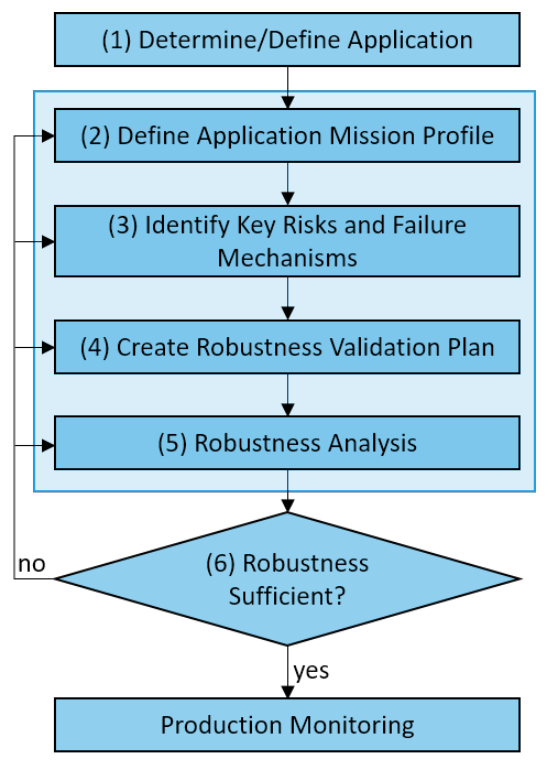

Fig. 1: Simplified robustness validation procedure [6] 
machine. There are many aspects, which cause an increment to the damage of electric machines. For example electrical aging, thermal aging, thermal-mechanical aging or thermal cycling are the main impact factors for the insulation system of electric machines [3]. On the mechanical side, especially the bearings are likely to fail. This is due to bearing currents or vibrations [7]. For a low voltage machine the winding system is the weakest spot. Most likely is the failure due to thermal aging in the stator windings in case the design excludes partial discharge [8].

To predict the thermal aging of machines, we need a precise knowledge of the conditions in which the machine will be operated. For aircraft this is the load, which the machine will face during a mission and over their lifetime. The load is in our use case the torque on the rotor shaft and is dependent on the thrust produced by the aircraft. Predominant losses in the machine are the ohmic losses in the copper windings, which will have the highest influence on the temperature. Equation (2)-(7) shows that the motor power $P_{\text {Mot }}$ is proportional to Torque $M_{\mathrm{Mot}}$ and this is proportional to the current $I$ in the stator winding. Most likely permanent magnet synchronous machines (PMSM) with surface magnets will be used in aircraft as propulsion motors. These motors do not need a field weakening region, because of the cubic dependency of the Power $P_{\text {Prop }}$ and the rotational speed $n_{\text {Prop }}$ (1). For this kind of machine, the inductance $L_{\mathrm{d}}$ and $L_{\mathrm{q}}$ are equal and therefore only the current in q-direction $i_{\mathrm{q}}$ will generate torque. The losses in the winding $P_{\mathrm{V}}$ are proportional to $I^{2}$ (5) and proportional to the temperature difference $\Delta \vartheta$ (6), which leads to the proportional coherence between $M_{\mathrm{Mot}}$ and $\sqrt{\Delta \vartheta}$ (7). This is the reason why a precise mission profile is needed in the beginning of the design process. We consider a constant ambient temperature, the amount of energy which can be dissipated raises with $\Delta \vartheta$ and is therefore linear as well. In a first assumption, the temperature is mainly dependent on the torque (7).

$$
\begin{aligned}
& P_{\text {Prop }} \sim n_{\text {Prop }}^{3} \\
& P_{\text {Mot }}=\frac{P_{\text {Prop }}}{\eta_{\text {Prop }}} \rightarrow P_{\text {Mot }} \sim P_{\text {Prop }} \\
& P_{\mathrm{Mot}}=2 \cdot \pi \cdot n_{\mathrm{Mot}} \cdot M_{\mathrm{Mot}} \rightarrow P \sim M \\
& M_{\mathrm{Mot}}=\frac{3}{2} \cdot p \cdot \psi_{\mathrm{PM}} \cdot i_{\mathrm{q}} \rightarrow M_{\mathrm{Mot}} \sim I \\
& P_{\mathrm{V}}=R \cdot I^{2} \rightarrow P_{\mathrm{V}} \sim I^{2} \\
& \Delta P_{\mathrm{V}}=m \cdot c \cdot \Delta \vartheta \rightarrow \Delta P_{\mathrm{V}} \sim \Delta \vartheta \\
& M \sim I \sim \sqrt{\Delta P_{\mathrm{V}}} \sim \sqrt{\Delta \vartheta}
\end{aligned}
$$

To design a machine, which fits to the requirements of a hybrid-electric aircraft, we need to find a solution to generate the requested data. This is in a first step the mission profile. Since today, no regional aircraft with a hybrid electric propulsion system is known, other ways needs to be taken. This leads to our approach to generate the mission profile with the web available data.

\section{Flight Mechanics}

The equations of motion (8) and (9) are established in relation to the system proposed in [9]. Therein $W$ denotes the weight load of the aircraft, $T$ denotes the thrust, $D$ describes the drag and $L$ describes the lift. The thrust angle $\alpha_{\mathrm{T}}$ is small for conventional aircraft and we assume that $\cos \left(\alpha_{\mathrm{T}}\right)=1$. Newton's second law of motion implies that the derivation of the airspeed $V$ multiplied with the mass $m$ is the accelerating force in longitudinal direction. In Z-direction the derivation of the flight-path angle $\gamma$ is used to describe the centripetal acceleration. In case of a constant flight in calm atmosphere these equations simplify to (10) and (11) with $\frac{\mathrm{d} V}{\mathrm{~d} t}=\frac{\mathrm{d} \gamma}{\mathrm{d} t}=0$ and $\alpha_{\mathrm{T}}=0$.

For flights during cruising phase, we calculate the characteristic values for the lift coefficient $C_{L}(11), q_{\infty}$ describes the dynamic pressure and $S$ the surface area of the wings. Further, we are able to calculate the drag coefficent $C_{\mathrm{D}}$ in (12). $C_{D_{0}}$ is the zero-lift drag, $C_{D_{\mathrm{i}}}$ describes the induced drag, which is lift dependent. The aspect ratio $A$ and the Oswald factor $e$ are aircraft specific coefficients [4] [9].

$$
\begin{gathered}
\sum F_{\mathrm{X}}=\frac{W}{g} \cdot \frac{\mathrm{d} V}{\mathrm{~d} t}=T \cdot \cos \left(\alpha_{\mathrm{T}}\right)-D-W \cdot \sin (\gamma) \\
\sum F_{\mathrm{Z}}=-\frac{W}{g} \cdot V \cdot \frac{\mathrm{d} \gamma}{\mathrm{d} t} \\
=-T \cdot \sin \left(\alpha_{\mathrm{T}}\right)-L+W \cdot \cos (\gamma) \\
T=D=C_{\mathrm{D}} \cdot q_{\infty} \cdot S \\
W=L=C_{\mathrm{L}} \cdot q_{\infty} \cdot S \\
C_{\mathrm{D}}=C_{\mathrm{D}_{0}}+D_{D_{\mathrm{i}}}=C_{\mathrm{D}_{0}}+\frac{C_{\mathrm{L}}^{2}}{\pi \cdot A \cdot e}
\end{gathered}
$$

In the special case of an aircraft with an all electric propulsion system, the weight load is not changing during the flight. For a hybrid-electric propulsion system this assumption is wrong, if kerosene or another fuel is burned. An estimation is difficult, because the level of hybridization is not known. However, it describes a worst case scenario. Therefore, we assume that the weight during the mission is constant.

The aerodynamic efficiency is described with the relation of $\frac{L}{D}$. The efficiency is kept constant for one flight. With this assumption, it is possible to solve (8) and (9) for any flight situation. The power requirement can be calculated by (13).

$$
P=T \cdot V
$$

\section{Flight data}

Table I shows a sample of the data available for all flights we tracked. For our calculations time stamp, altitude and speed are used. However, depending on the flight, in some cases time on the airport field is included. Thus, flight data need to be carefully processed and checked before the power calculation. Otherwise the actual mission time will not be found. 
TABLE I: Example of raw data

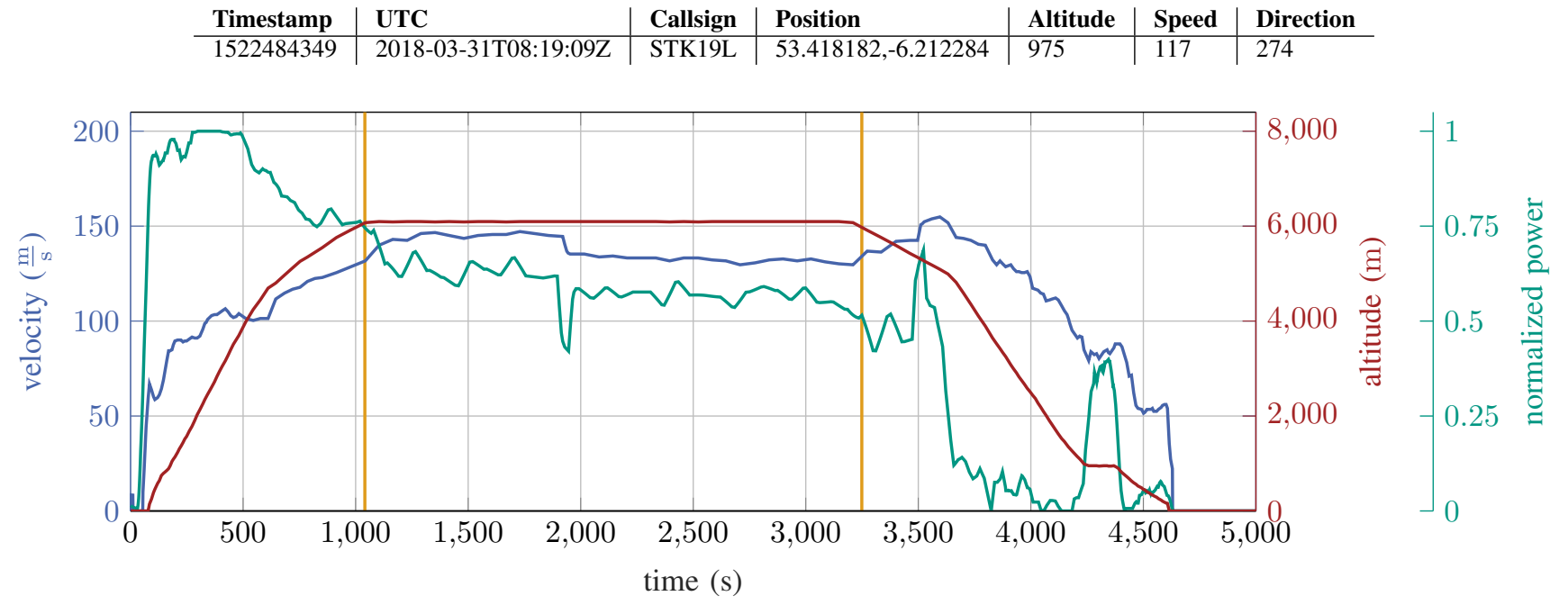

Fig. 2: Results of one flight analysis. On the $x$-axis the flight time, on the $y$-axis in blue the aircraft velocity, in red the aircraft altitude and in green the normalized power.

\section{E. Aircraft}

The most efficient type of aircraft is the turbo prop [10]. These aircraft use the benefit of the efficient propulsion system compared to a jet engine [10]. The drawback is that the actual traveling speed is lower compared to an aircraft with jet engine. Only two types of regional aircraft with a turbo prop are widely spread on the market. The ATR 72-600, produced by Avions de Transport Régional (ATR) and the DASH8 Q-400, produced by Bombardier. These two aircraft accommodate 70 seats and are of almost equal dimensions [11]. Due to a limited data access it is only possible to analyze the data of one type of aircraft to generate a reasonable amount of flight data in a limited period of time. This aircraft has a maximum reach of $1,185 \mathrm{~km}-1,400 \mathrm{~km}$ depending on the actual configuration. In our calculations we assume a maximum weight of $23,000 \mathrm{~kg}$ and a maximum power per engine of 2,051 kW. On the aircraft two engines are installed. Further details of the aircraft can be found in [11].

\section{RESUlts}

\section{A. Flight evaluation}

We use the method proposed in section II-C, to evaluate the flight data. The data mining was going on for 227 days and in that time 6,084 flights have been evaluated. This leads to the results shown in Fig. 2 for a single flight. The flight can be divided into the three different flight phases take-off and climb, cruise, descend and landing. In Fig. 2, the starting and ending points of these phases are marked with orange lines. The take-off and climb phase starts at 0 until 1040 seconds. The descend and landing phase lasts till the end of the flight. In the first flight phase, we see a rise in power and velocity of the aircraft. After reaching take-off speed at $60 \frac{\mathrm{m}}{\mathrm{s}}$, the aircraft starts to climb until it reaches the cruising altitude. We can observe a high demand in power, especially in the first climb phase. Afterwards the climb rate of the aircraft decreases and the speed rises. After reaching the cruising altitude, which is around $6000 \mathrm{~m}$ above sea level for this specific flight, the first phase ends and the cruise phase begins. The characteristics of the cruise phase is a constant power requirement. Only little adjustments are made due to minor speed changes or small changes in the travel height. In this case, we can observe a little drop in the power request during the flight, due to a change in cruising speed. The last flight phase is the descend and landing procedure. In the shown flight, we see an acceleration of the aircraft and a small negative climb rate in the beginning. Therefore, the power request is not dropping sharply. It just drops after the climb rate decreases and the decelerating begins. As we can see in this flight, we often recognize peaks in the power request, due to acceleration or continuous flight at the same height. Afterwards, the usual landing procedure starts and the power request is rather low.

In some flights the height is adjusted during the cruise phase. We separated that flight phase from the three main phases because the power request is higher. We call this phase the climb during cruise phase.

\section{B. Evaluation}

Evaluating the data of all flights shows that the average flight lasts one hour and 3 minutes and splits up into 18 minutes and 30 seconds take-off and climb, 25 minutes cruise and 17 minutes and 50 seconds descend and landing. The distribution of the flight time is shown in Fig. 3a.

The power distribution in the different flight phases is shown in Fig. 4. During the take-off and climb phases there is a mean power request of $74 \%$. During the cruise phases the power request drops to $56.5 \%$ and during the descend and landing phases the power request drops further to a mean value of $19 \%$. Fig. $4 \mathrm{~d}$ shows the power distribution for the phase 
climb during cruise. This maneuver is appearing quite rarely in only $11 \%$ of all analyzed flights. The median duration of this maneuver is 105 seconds. The mean value would not be meaningful for that case, since it is not normally distributed. The mechanical energy used by the shaft per flight is shown in Fig. 3c. The median of these data set is $2270 \mathrm{kWh}$. The standard deviation is $583 \mathrm{kWh}$. The highest flight distance in the data set is more than $1200 \mathrm{~km}$, has a flight time of over 2 hours and 50 minutes and the energy consumption is more than $6000 \mathrm{kWh} .95 \%$ of all flights need less than $3200 \mathrm{kWh}$.

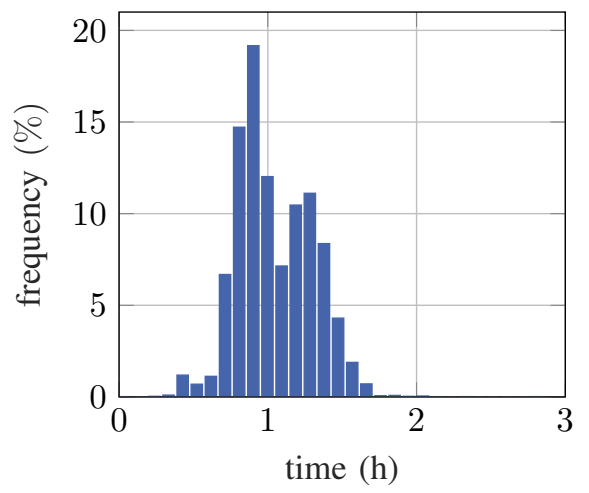

(a) Flight duration

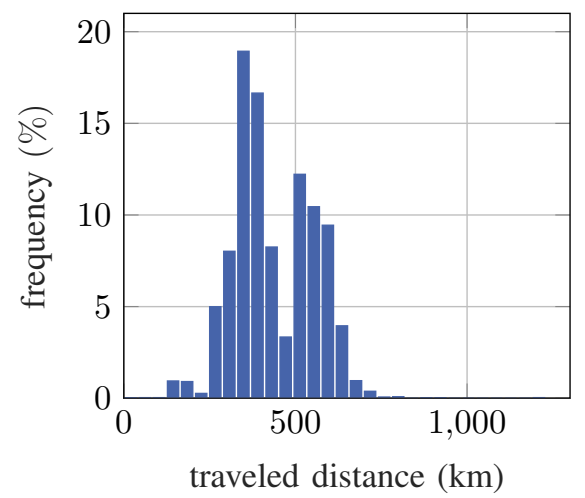

(b) Flight distance

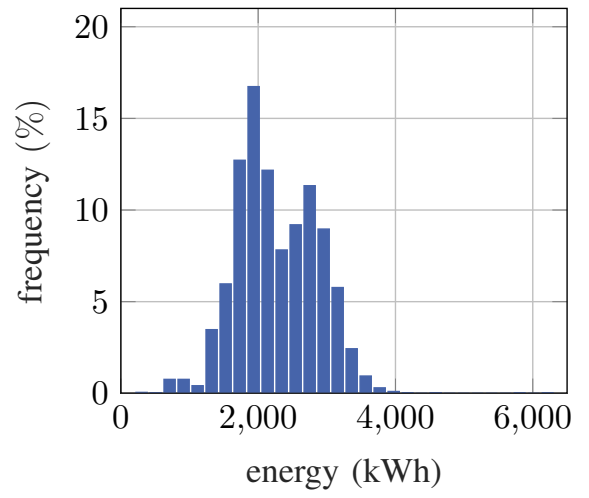

(c) Energy consumption

Fig. 3: Distributions of flight time, distance and energy consumption
The median flight has a distance of $407 \mathrm{~km}$. The standard deviation is $115 \mathrm{~km} .95 \%$ of all flights are shorter than $617 \mathrm{~km}$. The median flight duration is 59 minutes and 30 seconds. The standard deviation of the flight time is 15 minutes. $95 \%$ of all flights are shorter than 1 hour and 28 minutes. The median of the aircraft stopping at the ground is 39 minutes. In this data all stops are included, stop over night and longer stops, which were probably used for maintenance. The distributions are shown in Fig. 3a-c.

\section{Individual aircraft}

Table II shows the summary of the evaluated data for each aircraft. The traveled distance of the aircraft is between $575,000 \mathrm{~km}$ and $699,000 \mathrm{~km}$. The flight duration of each aircraft is close to each other, except for the aircraft EI-FNA, which has only 1,382 hours of flight duration and the least kilometers traveled. We assume that the gap is due to a major maintenance. The aircraft was out of service for 8.35 days which equals $200 \mathrm{~h}$. All aircraft have a median flight distance and maximum flight distance which is close to each other. Furthermore, all aircraft do operate on an average of 6 to 7 flights per day. This demonstrates that all data sets can be used to generate a specific mission profile for aircraft operating in that region.

\section{Mission profile}

Adding these findings together leads to the mission profile shown in Fig. 5. For the mission profile, we calculate a constant maximum power of 52 seconds in climb phase. The non constant power request is calculated with the power distribution and standard deviation. Fig. 4a shows the power distribution during the climb phase. The cruising phase is estimated as a constant power request. The descend and landing includes a power drop and a peak in power request.

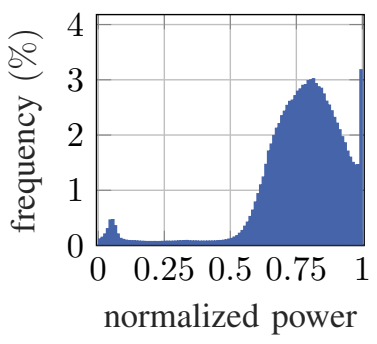

(a) Take-off and climb

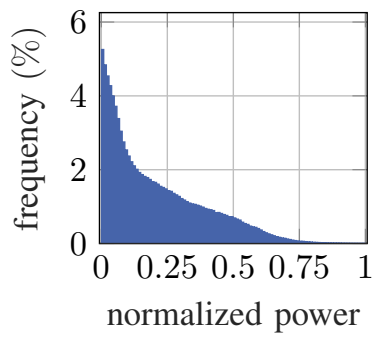

(c) Descend and landing

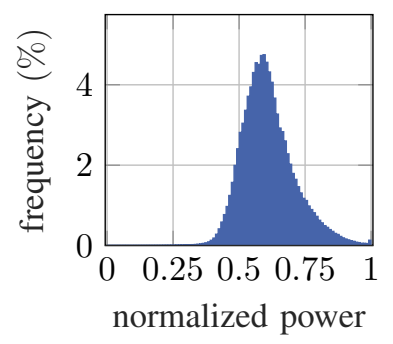

(b) Cruise

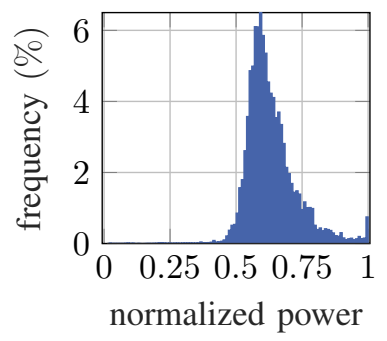

(d) Climb during cruise
Fig. 4: Power distribution during different flight phases 
TABLE II: Individual evaluation of aircraft

\begin{tabular}{l|l|l|l|l|l|l} 
Registration & $\begin{array}{l}\text { No. of } \\
\text { flights }\end{array}$ & $\begin{array}{l}\text { Max. time on } \\
\text { ground (in h) }\end{array}$ & $\begin{array}{l}\text { Distance } \\
\text { (in km) }\end{array}$ & $\begin{array}{l}\text { Flight time } \\
\text { (in h) }\end{array}$ & $\begin{array}{l}\text { Longest flight } \\
\text { (in km) }\end{array}$ & $\begin{array}{l}\text { Median flight } \\
\text { distance (in km) }\end{array}$ \\
\hline EI-FAS & 1551 & 73.4 & 680.000 & 1.613 & 905 & 402 \\
\hline EI-FAT & 1515 & 67.9 & 691.000 & 1.640 & 870 & 439 \\
\hline EI-FAV & 1575 & 50.8 & 699.000 & 1.672 & 764 & 418 \\
\hline EI-FNA & 1407 & 200.6 & 575.000 & 1.382 & 1.218 & 383
\end{tabular}

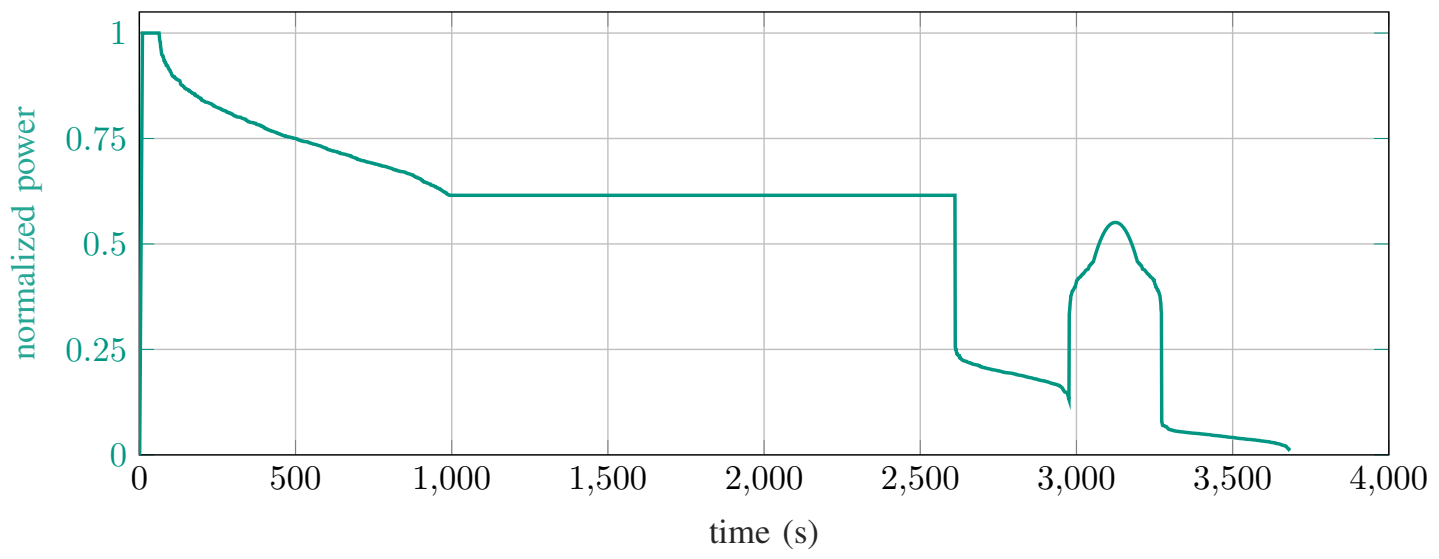

Fig. 5: Results summarized as mission profile for hybrid-electric regional aircraft

The descend and landing phase is seperated into three parts. First part is the section after the aircraft is leaving the constant travel height. The power requirement in that phase is usually between $10 \%$ and $30 \%$. The average time for that flight maneuver is 6 minutes and 5 seconds. Furthermore, the last raise in power is due to a time frame in which the aircraft accelerates or stays at the same altitude. This leads to a raise in power, which is above $30 \%$ of the nominal power. The last phase is the actual landing phase, in which the aircraft approaches the ground. The power request is below $10 \%$.

\section{E. Temperature load}

Taking the relations of (1) and (2), we calculate the torque and speed distribution of the machines in the aircraft, which are shown in Fig. 6. For that estimation, we assume that the pitch angle of the propeller is not adjustable and the motor speed is adjusted. Because of the cubic relation between power and rotational speed, the appearance of high rotational speed of more then $70 \%$ of the nominal speed is occuring in almost $75 \%$ of the flight time. The overall torque demand in is lower. With the torque demand, we are able to calculate a temperature distribution. This distribution assumes that the thermal time constant is zero and $\Delta \vartheta$ is immediately changing to the stationary temperature difference. Most of the time we face a temperature difference around $50 \%$ of the maximum temperature difference. Assuming that the allowed temperature difference between the stator winding $T_{\max \text {,winding }}=180^{\circ} \mathrm{C}$ and the ambient temperature $T_{\text {ambient }}=40{ }^{\circ} \mathrm{C}$ is around $140 \mathrm{~K}$, we can estimate that a motor, which reaches the maximum $\Delta \vartheta$ at maximum torque will be operated most of the time at around $110^{\circ} \mathrm{C}$.

\section{Discussion}

We look at a very small amount of aircraft in only one specific region. For these aircraft, we can show flight patterns and find a mission profile. To make sure that the found mission profile is valid for regional aircraft operating all over the world, more flights of different aircraft must be analyzed. This leads to the problem that coverage by ADS-B receivers is currently not good enough in remote regions. We compared our profile with the mission profile in [4]. Both data sets fit well. One of the differences is that our mission profile is missing the acceleration of the aircraft, at the end of the climbing phase. In the single evaluated flights, we sometimes see the specific

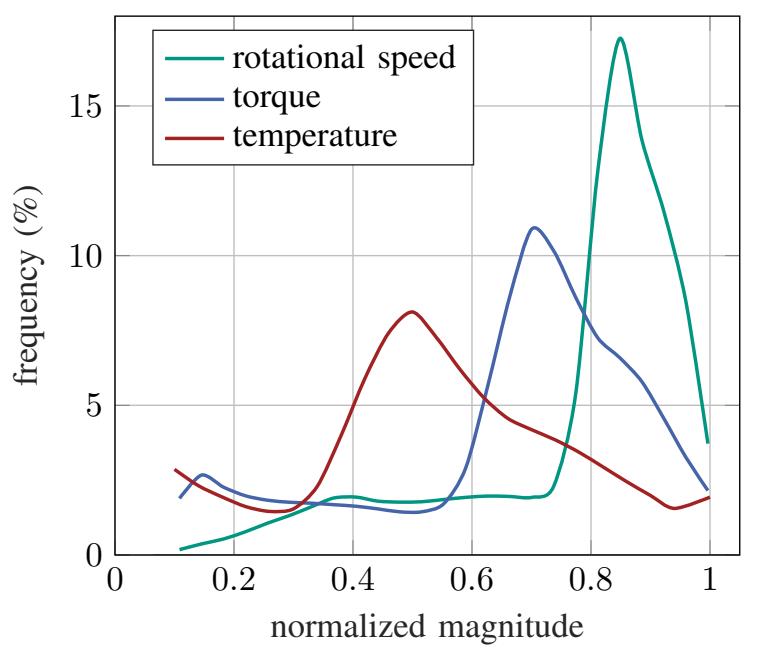

Fig. 6: Propability distribution of rotational speed, torque and temperature 
rise in power. We have not found a pattern yet, which allows us to incorporate said rise in power into the standard mission profile. This might be due to the quality of raw data. The recorded data has no constant time stamp and varies in a range of a few seconds up to 60 seconds. Thus, small changes in speed or height during flight are not documented. Further simplifications are made. We left out the phase climb during cruise, due to a limited occurrence in the data. This should not be neglected during the design process and needs to be evaluated in the specific design case. In terms of the linear damage accumulation, we should check if the occurrence in $10 \%$ of the flights delivers a reasonable amount of damage compared to the overall damage. The aircraft operational range is in the most time in a range of below $600 \mathrm{~km}$. Compared to the possible distance of up to $1400 \mathrm{~km}$, we see that the capability in the case of these aircraft is not needed. Especially in the design of the battery, weight will be the most dominant factor. Therefore the safety margin should be chosen as small as possible, to carry just as much weight as needed, but still fulfill the mission and the demand of the legislation. Further, the evaluation about the temperature distribution just delivers an idea of how high the motor temperature will be. This needs to be clarified on a specific motor design. We will look into that in our future work.

\section{CONCLUSION}

We are able to calculate a mission profile for a hybridelectric regional aircraft. The power requirement is calculated through basic equations of motion for flight dynamics and is based on real time flight data. The presented mission profile shows the normalized shaft power requirement of the aircraft during an average flight. This paper delivers the input for the second step of a robust design process for hybrid-electric regional aircraft. Based on the results, stress analysis can be performed for drivetrain components.

\section{REFERENCES}

[1] D. P. Raymer, Aircraft Design: A Conceptual Approach, 5th ed., ser. AIAA education series. Reston, VA: American Institute of Aeronautics and Astronautics, 2012.

[2] G. C. Montanari, G. Mazzanti, and L. Simoni, "Progress in electrothermal life modeling of electrical insulation during the last decades," IEEE Transactions on Dielectrics and Electrical Insulation, vol. 9, no. 5, pp. 730-745, Oct. 2002.

[3] V. I. J. Kokko, "Electrical ageing in lifetime estimation of hydroelectric generator stator windings," in The XIX International Conference on Electrical Machines - ICEM 2010, Sep. 2010, pp. 1-5.

[4] J. Thauvin, G. Barraud, X. Roboam, B. Sareni, M. Budinger, and D. Leray, "Hybrid propulsion for regional aircraft: A comparative analysis based on energy efficiency." IEEE, Nov. 2016, pp. 1-6.

[5] W. R. Richards, K. O'Brien, and D. C. Miller, "New Air Traffic Surveillance Technology," Aero/Boeing, vol. 02/2010, pp. 6-13, 2010.

[6] "Handbook for Robustness Validation of Semiconductor Devices in Automotive Applications," 2015.

[7] S. Chen and T. A. Lipo, "Bearing currents and shaft voltages of an induction motor under hard- and soft-switching inverter excitation," IEEE Transactions on Industry Applications, vol. 34, no. 5, pp. 10421048, Sep. 1998.

[8] F. Kielmann and M. Kaufhold, "Evaluation analysis of thermal ageing in insulation systems of electrical machines - a historical review -," IEEE Transactions on Dielectrics and Electrical Insulation, vol. 17, no. 5, pp. 1373-1377, Oct. 2010.
[9] E. Torenbeek and H. Wittenberg, Flight Physics: Essentials of Aeronautical Disciplines and Technology, with Historical Notes. Dordrecht; London: Springer, 2009.

[10] R. Babikian, S. P. Lukachko, and I. A. Waitz, "The historical fuel efficiency characteristics of regional aircraft from technological, operational, and cost perspectives," Journal of Air Transport Management, vol. 8, no. 6, pp. 389-400, Nov. 2002.

[11] P. D. Vecchia, "Development of Methodologies for the Aerodynamic Design and Optimization of New Regional Turboprop Aircraft," p. 229, 2013. 